
\title{
$\mathrm{AIP} \mid \begin{aligned} & \text { Physics of } \\ & \text { Fluids }\end{aligned}$
}

\section{Long nonlinear waves in an unbounded rotating jet or rotating twofluid flow}

S. M. Sun

Citation: Physics of Fluids (1994-present) 6, 1204 (1994); doi: 10.1063/1.868442

View online: http://dx.doi.org/10.1063/1.868442

View Table of Contents: http://scitation.aip.org/content/aip/journal/pof2/6/3?ver=pdfcov

Published by the AIP Publishing

\section{Articles you may be interested in}

Envelope equation of electrostatic nonlinear waves in relativistic two-fluid plasmas

Phys. Plasmas 17, 082310 (2010); 10.1063/1.3474954

Two-fluid jets and wakes

Phys. Fluids 16, 1037 (2004); 10.1063/1.1651481

A nonlinear two-fluid model for toroidal plasmas

Phys. Plasmas 7, 4644 (2000); 10.1063/1.1308083

Envelope equation for nonlinear transverse waves in a warm two-fluid plasma

Phys. Plasmas 5, 3853 (1998); 10.1063/1.873105

Nonlinear waves in twofluid hydrodynamics

Phys. Fluids B 5, 2062 (1993); 10.1063/1.860793

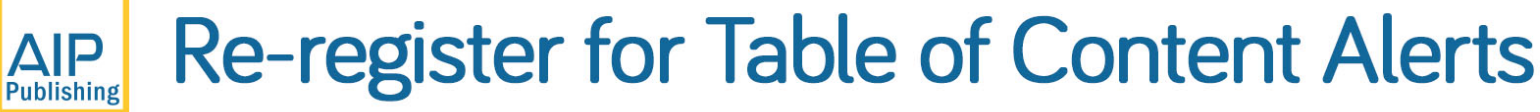




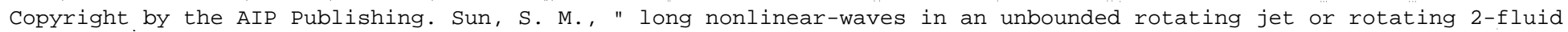
flow," Phys. Fluids 6, 1204 (1994); http://dx.doi.org/10.1063/1.868442

\title{
Long nonlinear waves in an unbounded rotating jet or rotating two-fluid flow
}

\author{
S. M. Sun \\ Department of Mathematics, Virginia Polytechnic Institute and State University, Blacksburg, Virginia \\ 24061
}

(Received 9 April; accepted 5 November 1993)

\begin{abstract}
The objective of this paper is to study weakly nonlinear waves in an infinitely long rotating jet and a rotating two-fluid flow bounded by an infinitely long rigid cylinder with surface tension at the interface. The critical values for Rossby number, a nondimensional wave speed, are found. When the Rossby number is near one of the critical values, nonlinear theory is developed under long-wave approximation and the well-known Korteweg-de Vries (KdV) equations for the free surface and free interface are obtained. Then the solitary wave solutions are given as the first-order approximations of the solutions of the equations governing the motion of the flows. The analogy between the rotating fluid flows and a two-dimensional flow with density stratification is discussed.
\end{abstract}

\section{INTRODUCTION}

The motion of a rotating fluid has been studied for a long time. In the 18th century, d'Alembert already knew that the mass-times-acceleration term in Newton's second law can be moved to the other side of the equation and be considered as a body force acting in the opposite direction to the acceleration. In a rotating system, the centrifugal force and Coriolis force are the body forces to replace the usual gravitational force or other forces. This is attractive in many contexts, for example, see Greenspan, ${ }^{1}$ and is compared to many other fluids, such as stratified fluids. ${ }^{2} \mathrm{~A}$ particular problem is the wave motion in a long rotating axisymmetric flow of an inviscid fluid with constant density bounded by a cylinder. Long ${ }^{3}$ studied this problem and gave the form of general solutions for large Rossby number, a nondimensional wave speed, if a uniform rotating flow is imposed at infinity, while Yih et al. ${ }^{4}$ also considered the problem and gave a formal solution when the Rossby number is smaller. The internal waves in a rotating fluid with arbitrary radial shear and radially dependent angular speed at infinity have been studied intensively within 40 years, since it was suggested that the model can be used to explain the fundamental nature of the vortex breakdown, which has frequently been observed to occur in the flow fields of slender delta wings. Many experiments ${ }^{5,6}$ and theoretical studies $^{7-9}$ have been done since the initial development by Squire. ${ }^{10} \mathrm{~A}$ detailed review can be found in the paper by Leibovich. ${ }^{11}$

This paper concerns weakly nonlinear waves at the free surface of an infinitely long rotating jet and the interface of a rotating two-fluid flow in an infinitely long cylinder. These two problems have many physical applications. As pointed out by Berger, ${ }^{12}$ the vortex breakdown should be viewed as a free boundary problem rather than a rotating fluid bounded by a rigid cylindrical boundary. The vorticity is confined to a bounded region in the radial direction, called the vorticity core. The vorticity core should be surrounded by the irrotational part of the fluid outside. Therefore to determine the wave motion inside the vorticity core it is more realistic to study a long rotating jet with a free boundary. This is also true in studying some meteorological motions, such as the formation of tornadoes. ${ }^{13}$ The wave behavior of liquid columns in zero-gravity manufacturing processes, as, for example, crystal growth process in an orbiting space laboratory, is another interesting application. In such a free floating zone, the so-obtained crystals exhibit enhanced electronical qualities and large aspect ratios. It has been found that a rotating cylindrical column can stabilize the production process of a perfect crystal. ${ }^{14}$ A linear stability analysis was conducted by Bauer ${ }^{15,16}$ using inviscid liquid model and by Gillis and Shuh ${ }^{17}$ and Gillis and Kaufmann ${ }^{18}$ using the viscous liquid model. However, for large-amplitude waves nonlinear theory is necessary to study the motion of such liquids. The twolayer rotating flow is useful in a process of separation of a fluid mixture in a centrifugal force field. By assuming that the mixture consists of two fluids, as, for example, oil and water, and initially the fluids occupy the interior of a long cylinder in a solid body rotation, Greenspan ${ }^{19}$ and Ungarish ${ }^{20}$ have studied the transient process, leading to a final state of separated fluids. It is certainly of great interest to know the wave motions at the interface of the final state.

It has been long recognized that the stratification of circulation plays a similar role in a rotating fluid as the stratification of density in a stratified fluid. In order to have a nonlinear theory for rotating fluids, we first review the nonlinear results for stratified fluids. If we only consider traveling waves and assume that the speed of the waves in horizontal direction is $c$, then a nondimensional wave speed $F=c /(g h)^{1 / 2}$ can be defined, which is called the Froude number, where $g$ is gravitational acceleration and $h$ is the depth of flow at infinity. For a fluid of constant density, $F=1$ is the only critical value of $F$ and for $F$ near unity the linear theory of the problem is not valid and nonlinear theory must be developed. Korteweg and $\mathrm{deV}$ ries $^{21}$ first used a long-wave approximation and derived a model equation for the surface elevation, which is now called the $\mathrm{KdV}$ equation and has a traveling wave solution called the solitary wave solution. Peters and Stoker ${ }^{22}$ stud- 
ied a two-layer fluid and a stratified fluid with free surface. It is found that for a two-layer fluid there are two critical values for $F$ and for a stratified fluid there exist infinitely many critical values of $F$, near each of which a $\mathrm{KdV}$ equation is derived and solitary waves are found. Further derivations of the $\mathrm{KdV}$ equation and the solitary waves in a stratified fluid by using more general asymptotic methods have also been obtained. ${ }^{23,24}$ Vanden-Broeck and Turner ${ }^{25}$ used numerical computation to find the solitary wave solutions of the exact equations for $F$ near the second critical value, while Akylas and Grimshaw ${ }^{26}$ used a formal method to study the same problem. A rigorous proof of existence of such solitary wave solutions for the exact equations was given by Ter-Krikorov ${ }^{27}$ for $F$ near the first critical value and Sun and Shen ${ }^{28,29}$ for $F$ near the first two critical values with surface tension.

The wave phenomena in a stratified fluid can also be obtained in a long rotating fluid. For a long rotating flow in a cylinder with continuously radial shear and radially dependent angular speed, the long wave theory can be developed and solitary waves have been found, ${ }^{8,9}$ which was confirmed by experiments. ${ }^{5,6}$ Recently, Grimshaw ${ }^{30}$ studied the flow of a rotating fluid past an obstacle placed on the axis of a cylinder and derived the forced $\mathrm{KdV}$ equation for the flow. However, if the upstream is a uniform rotating flow, the problem is linear and the nonlinear term in the equation is zero. We note that all the above cases do not include the one studied in the paper because of the discontinuity at the free surface or interface, although their methods can be applied using streamfunctions. Here we shall develop a nonlinear theory for a long rotating axisymmetric flow with a free boundary and a rotating two-fluid flow in a long cylinder. For both cases, the $\mathrm{KdV}$ equation for the deviation of the free surface or interface from the equilibrium state will be obtained under long-wave approximation from the exact Euler equations rather than the equations obtained using the streamfunctions. The solitary wave solutions will be obtained as the first-order approximations of the solutions of the exact equations. A rigorous proof of the existence of such solutions is underway and some difficulties are expected to arise in the proof when the small oscillatory tails of the solutions appear at infinity, which is a similar phenomenon for the solutions of stratified fluids. ${ }^{29}$

This paper is organized as follows. In Sec. II, a singlelayer rotating fluid bounded by a free surface is studied, and the $\mathrm{KdV}$ equation is derived under long-wave approximation. Then the solitary wave solution is obtained and the first-order approximation of the velocity and pressure is given. In Sec. III, a rotating two-fluid flow bounded by a rigid cylinder with a free interface between the fluids is considered and the solitary waves are derived. Some limiting cases are discussed. In Sec. IV, the results are summarized and some applications are mentioned.

\section{LONG WAVES IN A ROTATING AXISYMMETRIC JET}

We first consider an infinitely long rotating axisymmetric flow of an inviscid and incompressible fluid of constant

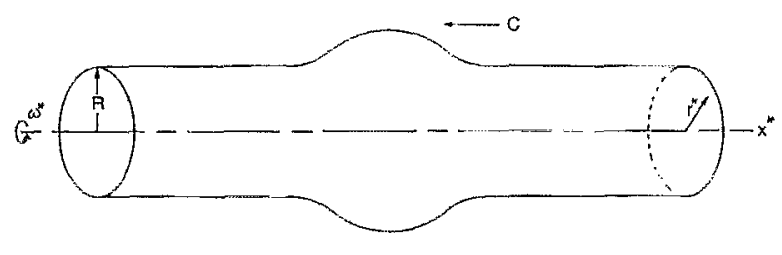

FIG. 1. A long rotating fluid with free surface.

density without surface tension on the free surface. Assume that there exist axisymmetric surface waves moving in the direction of the longitudinal axis of the flow (Fig. 1). In reference to a cylindrical coordinate system rotating with a constant angular velocity $\omega^{*}$, with an $x^{*}$ axis coincident with the axis of rotation and the cross section in the $r^{*}-\theta$ plane, the governing equations are the following: ${ }^{1}$ in $0<r^{*}<R+\eta^{*}\left(t^{*}, x^{*}\right),-\infty<x^{*}<+\infty$,

$$
\begin{aligned}
& \frac{\partial\left(r^{*} u^{*}\right)}{\partial r^{*}}+\frac{\partial\left(r^{*} w^{*}\right)}{\partial x^{*}}=0, \\
& \rho\left(\frac{D u^{*}}{D t^{*}}-\frac{\left(v^{*}\right)^{2}}{r^{*}}-2 \omega^{*} v^{*}-\left(\omega^{*}\right)^{2} r^{*}\right)=-\frac{\partial p^{*}}{\partial r^{*}}, \\
& \rho\left(\frac{D v^{*}}{D t^{*}}+\frac{u^{*} v^{*}}{r^{*}}+2 \omega^{*} u^{*}\right)=0, \\
& \rho \frac{D w^{*}}{D t^{*}}=-\frac{\partial p^{*}}{\partial x^{*}}
\end{aligned}
$$

on the boundary $r^{*}=\eta^{*}+R$,

$$
\begin{aligned}
& \frac{\partial \eta^{*}}{\partial t^{*}}+w^{*} \frac{\partial \eta^{*}}{\partial x^{*}}-u^{*}=0, \\
& p^{*}=P_{0}^{*},
\end{aligned}
$$

where $u^{*}\left(t^{*}, x^{*}, r^{*}\right), v^{*}\left(t^{*}, x^{*}, r^{*}\right), w^{*}\left(t^{*}, x^{*}, r^{*}\right)$ are used to denote the velocity components in the directions of $r^{*}, \theta$, and $x^{*}$, respectively, $p^{*}\left(t^{*}, x^{*}, r^{*}\right)$ is the pressure, $\rho$ is the density, $R+\eta^{*}\left(t^{*}, x^{*}\right)$ is the free surface, $P_{0}^{*}$ is the constant pressure outside the flow, and

$$
\frac{D}{D t^{*}}=\frac{\partial}{\partial t^{*}}+u^{*} \frac{\partial}{\partial r^{*}}+w^{*} \frac{\partial}{\partial x^{*}} .
$$

It is easy to find a uniform equilibrium state,

$$
\begin{aligned}
& u^{*}=0, \quad v^{*}=0, \quad w^{*}=c, \quad \eta^{*}=0, \\
& p^{*}=P_{0}^{*}+\left[\left(r^{*}\right)^{2}-R^{2}\right]\left(\omega^{*}\right)^{2} / 2,
\end{aligned}
$$

where $c$ is any real constant and is called wave speed of the flow, which is assumed here to be nonzero.

Since we are only interested in the existence of long waves in the fluid, we make the variables nondimensional by using appropriate units and applying a stretching transformation based upon the long wave approximation. Let $\epsilon$ be a small parameter representing the ratio of a radial length scale of the flow to a longitudinal length scale and define the nondimensional variables as 


$$
\begin{aligned}
& (r, x)=\left(r^{*}, \epsilon^{1 / 2} x^{*}\right) / R, \quad t=2 \epsilon^{3 / 2} \omega^{*} t^{*}, \\
& \mathrm{Ro}=\frac{c}{\left(2 \omega^{*} R\right)}, \quad(u, v, w)=\frac{\left(\epsilon^{-1 / 2} u^{*}, v^{*}, w^{*}\right)}{\left(2 \omega^{*} R\right)}, \\
& p=\frac{p^{*}}{\left[4 \rho\left(\omega^{*} R\right)^{2}\right]}, \quad P_{0}=\frac{P_{0}^{*}}{\left[4 \rho\left(\omega^{*} R\right)^{2}\right]},
\end{aligned}
$$

where Ro is called the Rossby number and is a nondimensional wave speed. In terms of these variables, the governing equations (1) $-(6)$ become

$$
\begin{aligned}
& (r u)_{r}+(r w)_{x}=0, \\
& \epsilon^{2} u_{t}+\epsilon u u_{r}+\epsilon w u_{x}-r^{-1} v^{2}-v-(r / 4)=-p_{r}, \\
& \epsilon v_{t}+u v_{r}+w v_{x}+r^{-1} u v+u=0, \\
& \epsilon w_{t}+u w_{r}+w w_{x}=-p_{x} ;
\end{aligned}
$$

on the boundary $r=1+\eta$,

$$
\begin{aligned}
& \epsilon \eta_{t}+w \eta_{x}-u=0, \\
& p=P_{0} .
\end{aligned}
$$

A uniform equilibrium state of (7)-(12) is $u_{0}=0, \quad v_{0}=0, \quad w_{0}=$ Ro, $\quad \eta_{0}=0, \quad p_{0}=P_{0}+\left(r^{2}-1\right) / 8$.

To find nontrivial solutions of (7)-(12), we use the following form of the asymptotic expansion:

$$
f(t, x, r)=f_{0}(t, x, r)+\epsilon f_{1}(t, x, r)+\epsilon^{2} f_{2}(t, x, r)+\cdots,
$$

for $u, v, w, \eta$, and $p$, respectively, and $\mathrm{Ro}=\lambda+\sigma \epsilon$ for small $\epsilon$, where $u_{0}, v_{0}, w_{0}, \eta_{0}$, and $p_{0}$ are chosen as the equilibrium state (13). After substituting the asymptotic expansions of $u, v, w, \eta, p$ into (7)-(12), we can have a sequence of equations and boundary conditions for the successive approximations. The first-order approximation is

$$
\begin{aligned}
& \left(r u_{1}\right)_{r}+r w_{1 x}=0, \\
& v_{1}-p_{1 r}=0, \\
& u_{1}+\lambda v_{1 x}=0, \\
& p_{1 x}+\lambda w_{1 x}=0 ;
\end{aligned}
$$

at $r=1$,

$$
\begin{aligned}
& u_{1}-\lambda \eta_{1 x}=0, \\
& p_{1}+\left(\eta_{1} / 4\right)=0 .
\end{aligned}
$$

This is a nonstandard eigenvalue problem and $\lambda$ is the eigenparameter. By separation of variables and assuming that $u_{1}, v_{1}, \eta_{1}$, and $p_{1}$ are zero and $w_{1}=\sigma$ at infinity, we can obtain that $\lambda$ satisfies

$$
J_{1}(1 / \lambda)+4 \lambda J_{0}(1 / \lambda)=0,
$$

and

$u_{1}=\eta_{1 x}(t, x)\left(\frac{-J_{1}(r / \lambda)}{4 J_{0}(1 / \lambda)}\right), \quad v_{1}=\eta_{1}(t, x)\left(\frac{J_{1}(r / \lambda)}{4 \lambda J_{0}(1 / \lambda)}\right)$,

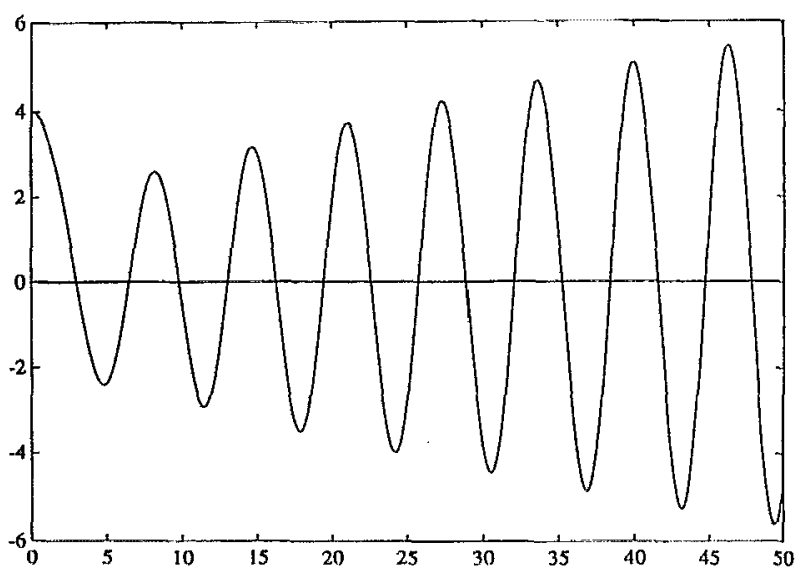

FIG. 2. The graph of function $\mu J_{1}(\mu)+4 J_{0}(\mu)$ for $0<\mu<50$.

$w_{1}=\sigma+\eta_{1}(t, x)\left(\frac{J_{0}(r / \lambda)}{4 \lambda J_{0}(1 / \lambda)}\right), \quad p_{1}=\eta_{1}(t, x)\left(\frac{-J_{0}(r / \lambda)}{4 J_{0}(1 / \lambda)}\right)$,

where $J_{n}(z), n=0,1$ are the Bessel functions of the first kind of order zero and unity. ${ }^{32}$ By using the asymptotic formulas $^{32}$ of $J_{0}(z)$ and $J_{1}(z)$, it is easy to show that there are infinitely many solutions of the equation $\mu J_{1}(\mu)+4 J_{0}(\mu)=0$, with the positive roots $0<\mu_{1}<\mu_{2}<\mu_{3}<\cdots \rightarrow+\infty$. Figure 2 gives the graph of $\mu J_{1}(\mu)+4 J_{0}(\mu)$, and also shows the first several roots of the function. Therefore $1 / \mu_{n}, n=1,2,3, \ldots$, are the critical values for $\lambda$. If $\lambda$ is chosen as $\lambda_{n}=1 / \mu_{n}$ for some $n>0$, then the solution given by (22) and (23) satisfies (15)-(20). However, $\eta_{1}(t, x)$ is still undetermined. To find $\eta_{1}$, the second-order approximation of the equations and boundary conditions must be used, which is the following:

$$
\begin{aligned}
& \left(r u_{2}\right)_{r}+\left(r w_{2}\right)_{x}=0, \\
& v_{2}-p_{2 r}=\lambda_{n} u_{1 x}-r^{-1} v_{1}^{2}, \\
& u_{2}+\lambda_{n} v_{2 x}=-v_{1 t}-u_{1} v_{1 r}-w_{1} v_{1 x}+r^{-1} u_{1} v_{1}, \\
& p_{2 x}+\lambda_{n} w_{2 x}=-w_{1 t}-u_{1} w_{1 r}-w_{1} w_{1 x}
\end{aligned}
$$

at $r=1$,

$$
\begin{aligned}
& u_{2}-\lambda_{n} \eta_{2 x}=\eta_{1 t}+w_{1} \eta_{1 x}-u_{1 r} \eta_{1}, \\
& p_{2}+\left(\frac{1}{4}\right) \eta_{2}=-\left(\frac{1}{8}\right) \eta_{1}^{2}-\eta_{1} p_{1 r} .
\end{aligned}
$$

This is a nonhomogeneous boundary value problem and $\lambda_{n}$ is an eigenvalue for corresponding homogeneous problem (15) $-(20)$. Therefore, to have a solution of (24) $-(29)$, the nonhomogeneous terms, which are the right-hand side of (24)-(29), must satisfy a solvability condition. From the solvability condition and a straightforward calculation, we can obtain the following equation for $\eta_{1}$ :

$$
A_{0} \eta_{1 t}+\sigma A_{0} \eta_{1 x}+A_{1} \eta_{1} \eta_{1 x}+A_{2} \eta_{1 x x x}=0,
$$

where 


$$
\begin{aligned}
& A_{0}=\left(\frac{1}{2 \lambda_{n}^{2}}\right)\left\{\left[\left(\frac{4}{J_{1}^{2}\left(1 / \lambda_{n}\right)}\right)\right] \int_{0}^{1} J_{1}^{2}\left(\frac{r}{\lambda_{n}}\right) r d r-1\right\}, \\
& A_{1}=-\left(\frac{3}{\lambda_{n}}\right)\left[1+\left(\frac{1}{16 \lambda_{n}^{2}}\right)\right]<0, \\
& A_{2}=-\left[\left(\frac{\lambda_{n}}{J_{1}^{2}\left(1 / \lambda_{n}\right)}\right)\right] \int_{0}^{1} J_{1}^{2}\left(\frac{r}{\lambda_{n}}\right) r d r<0 .
\end{aligned}
$$

By using (21) and the properties of Bessel functions, it can be shown that $A_{0}>0$ for all $n>1$.

Therefore (30) can be rewritten as

$$
\eta_{1 t}+\sigma \eta_{1 x}+\left(A_{1} / A_{0}\right) \eta_{1} \eta_{1 x}+\left(A_{2} / A_{0}\right) \eta_{1 x x x}=0 .
$$

This is a well-known $\mathrm{KdV}$ equation and possesses traveling wave solutions with the following form:

$$
\eta_{1}(t, x)=f[x-(\sigma+h) t],
$$

where $f(y)$ satisfies

$$
-h f_{y}+\left(A_{1} / A_{0}\right) f f_{y}+\left(A_{2} / A_{0}\right) f_{y y y}=0 .
$$

This equation has periodic solutions, so-called cnoidal wave solutions, and, as a limiting case, it also possesses a solitary wave solution,

$$
f(y)=\left(3 A_{0} h / A_{1}\right) \operatorname{sech}^{2}\left[\left(A_{2} h / 4 A_{0}\right)^{1 / 2} y+\delta\right],
$$

which implies

$$
\begin{aligned}
\eta_{1}(t, x)= & \left(3 A_{0} h / A_{1}\right) \operatorname{sech}^{2}\left\{\left(A_{2} h / 4 A_{0}\right)^{1 / 2}\right. \\
& \times[x-(\sigma+h) t]+\delta\},
\end{aligned}
$$

where $h$ and $\delta$ are real constants and $h$ is negative since $A_{0}$ is positive and $A_{2}$ is negative.

In what follows, we shall confine our attention to the steady waves with $h=-\sigma, \delta=0$ in (32) to have steady solitary wave solution,

$$
\eta_{1}(x)=\left(-3 \sigma A_{0} / A_{1}\right) \operatorname{sech}^{2}\left[\left(-\sigma A_{2} / 4 A_{0}\right)^{1 / 2} x\right],
$$

where $\sigma$ is positive, which implies that the steady solitary waves are always supercritical for all $n>1$ and only solitary waves of elevation exist. Then we can have the first-order approximation of the solution $u_{1}, v_{1}, w_{1}, \eta_{1}$, and $p_{1}$ from (22) and (23). This procedure can be used to find any higher-order approximation of the solutions of (7)-(12), which will not be carried out further here. If we return to the dimensional variables for the solitary wave solutions of $(1)-(6)$, we can collect the results in the following way:

$$
\begin{aligned}
u^{*}\left(x^{*}, r^{*}\right)= & 2 \omega^{*} R \epsilon^{3 / 2}\left(\lambda_{n} \frac{J_{1}\left(r^{*} / \lambda_{n} R\right)}{J_{1}\left(1 / \lambda_{n}\right)}\right)\left(\frac{3 \sigma}{A_{1}}\right) \\
& \times\left(-\sigma A_{0} A_{2}\right)^{1 / 2} \operatorname{sech}^{2}\left(\frac{\left(-\epsilon \sigma A_{2} / 4 A_{0}\right)^{1 / 2} x^{*}}{R}\right) \\
& \times \tanh \left(\frac{\left(-\epsilon \sigma A_{2} / 4 A_{0}\right)^{1 / 2} x^{*}}{R}\right)+O\left(\epsilon^{2}\right),
\end{aligned}
$$

$$
\begin{aligned}
v^{*}\left(x^{*}, r^{*}\right)= & -2 \omega^{*} R \epsilon\left(\frac{J_{1}\left(r^{*} / \lambda_{n} R\right)}{J_{1}\left(1 / \lambda_{n}\right)}\right)\left(\frac{-3 \sigma A_{0}}{A_{1}}\right) \\
& \times \operatorname{sech}^{2}\left(\frac{\left(-\epsilon \sigma A_{2} / 4 A_{0}\right)^{1 / 2} x^{*}}{R}\right)+O\left(\epsilon^{2}\right), \\
w^{*}\left(x^{*}, r^{*}\right)= & 2 \omega^{*} R\left[\lambda_{n}+\epsilon\left(\sigma-\frac{J_{0}\left(r^{*} / \lambda_{n} R\right)}{J_{1}\left(1 / \lambda_{n}\right)}\right)\right]\left(\frac{-3 \sigma A_{0}}{A_{1}}\right) \\
& \times \operatorname{sech}^{2}\left(\frac{\left(-\epsilon \sigma A_{2} / 4 A_{0}\right)^{1 / 2} x^{*}}{R}\right)+O\left(\epsilon^{2}\right), \\
p^{*}\left(x^{*}, r^{*}\right)= & P_{0}^{*}+\left(\omega^{*}\right)^{2} \frac{\left[\left(r^{*}\right)^{2}-R^{2}\right]}{2}+\left[4 \rho\left(\omega^{*} R\right)^{2} \epsilon\right] \\
& \times\left(\frac{\lambda_{n} J_{0}\left(r^{*} / \lambda_{n} R\right)}{J_{1}\left(1 / \lambda_{n}\right)}\right)\left(\frac{-3 \sigma A_{0}}{A_{1}}\right) \\
& \times \operatorname{sech}^{2}\left(\frac{\left(-\epsilon \sigma A_{2} / 4 A_{0}\right)^{1 / 2} x^{*}}{R}\right)+O\left(\epsilon^{2}\right), \\
\eta^{*}\left(x^{*}\right)= & R \epsilon\left(\frac{-3 \sigma A_{0}}{A_{1}}\right) \\
\times & \operatorname{sech}^{2}\left(\frac{\left(-\epsilon \sigma A_{2} / 4 A_{0}\right)^{1 / 2} x^{*}}{R}\right)+O\left(\epsilon^{2}\right) .
\end{aligned}
$$

Here $\lambda_{n}=1 / \mu_{n}$ is defined in (21) and is a critical Rossby number. From the first-order approximation of the solution, the wave profile at the free surface is given by

$$
\begin{aligned}
r^{*}= & R+\eta^{*}\left(x^{*}\right) \\
= & R+R \epsilon\left(\frac{-3 \sigma A_{0}}{A_{1}}\right) \\
& \times \operatorname{sech}^{2}\left(\frac{\left(-\epsilon \sigma A_{2} / 4 A_{0}\right)^{1 / 2} x^{*}}{R}\right)+O\left(\epsilon^{2}\right) .
\end{aligned}
$$

For $n \geqslant 2, J_{1}\left(r^{*} / \lambda_{n} R\right)$ has its maximum value in $0<r^{*}<R$. Thus the largest displacement of the solitary waves from the equilibrium state is not at the free surface, but in the fluid, which are internal solitary waves.

We conclude this section by comparing the fluid flow in a rotating frame of reference with the flow of a stratified fluid. From the first-order approximation, which is essentially the linear theory of the problem, there exist infinitely many critical speeds $\lambda_{n}, n=1,2, \ldots$, for the existence of the solitary waves, which is similar to the case of a stratified fluid. $^{22}$ When we use the nonlinear theory for both the rotating fluid and stratified fluid, the KdV equations can also be derived under the long-wave approximation. From (30) and (31), the coefficients of the $\mathrm{KdV}$ equation will never become zero. But the coefficients of the corresponding $\mathrm{KdV}$ equation from a stratified fluid can be zero, and the stretching for the $\mathrm{KdV}$ equation in such a case is no longer valid and new stretching parameters must be introduced. $^{31}$ 


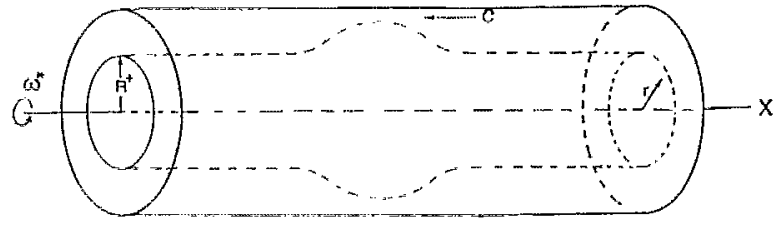

FIG. 3. A rotating two-fluid flow in a long cylinder.

\section{INTERNAL WAVES IN A TWO-FLUID FLOW}

In this section, we consider a long rotating flow of two immiscible, inviscid, and incompressible fluids of different but constant densities, with surface tension at the interface and bounded by a rigid cylinder (Fig. 3). Assume that the motion of the flow is axisymmetric and the density of the inner-layer fluid is $\left(\rho^{*}\right)^{+}$and the density of outer-layer fluid is $\left(\rho^{*}\right)^{-}$, with $\left(\rho^{*}\right)^{-}>\left(\rho^{*}\right)^{+}$, since there exists a centrifugal force in the rotating fluid. In reference to a cylindrical coordinate system rotating with a constant angular speed $\omega^{*}$ with the axis of the rotation as the $x^{*}$ axis and the cross section in the $r^{*}-\theta$ plane, the governing equations are the following: ${ }^{1}$ in $0 \leqslant r^{*}<R^{+}+\zeta^{*}$ or $R^{+}+\zeta^{*}<r^{*}<R^{-}$and $-\infty<x^{*}<+\infty$,

$$
\begin{aligned}
& \frac{\partial\left(r^{*} u^{*}\right)}{\partial r^{*}}+\frac{\partial\left(r^{*} w^{*}\right)}{\partial x^{*}}=0, \\
& \rho^{*}\left(\frac{D u^{*}}{D t^{*}}-\frac{\left(v^{*}\right)^{2}}{r^{*}}-2 \omega^{*} v^{*}-\left(\omega^{*}\right)^{2} r^{*}\right)=-\frac{\partial p^{*}}{\partial r^{*}}, \\
& \rho^{*}\left(\frac{D v^{*}}{D t^{*}}+\frac{u^{*} v^{*}}{r^{*}}+2 \omega^{*} u^{*}\right)=0, \\
& \rho^{*} \frac{D w^{*}}{D t^{*}}=-\frac{\partial p^{*}}{\partial x^{*}},
\end{aligned}
$$

and

$u^{*}, v^{*}, w^{*}, p^{*}, \rho^{*}=\left\{\begin{array}{c}\left(u^{*}\right)^{+},\left(v^{*}\right)^{+},\left(w^{*}\right)^{+},\left(p^{*}\right)^{+},\left(\rho^{*}\right)^{+}, \\ \text {for } 0<r^{*}<R^{+}+\zeta^{*} \\ \left(u^{*}\right)^{-},\left(v^{*}\right)^{-},\left(w^{*}\right)^{-},\left(p^{*}\right)^{-},\left(\rho^{*}\right)^{-}, \\ \text {for } R^{+}+\zeta^{*}<r^{*}<R^{-}\end{array}\right.$

on the interface $r^{*}=\zeta^{*}+R^{+}$,

$$
\begin{aligned}
& \frac{\partial \zeta^{*}}{\partial t^{*}}+\left(w^{*}\right)^{+} \frac{\partial \zeta^{*}}{\partial x^{*}}-\left(u^{*}\right)^{+}=0 \\
& \frac{\partial \zeta^{*}}{\partial t^{*}}+\left(w^{*}\right)^{-} \frac{\partial \zeta^{*}}{\partial x^{*}}-\left(u^{*}\right)^{-}=0 \\
& \left(p^{*}\right)^{-}-\left(p^{*}\right)^{+}=T S^{*}
\end{aligned}
$$

at the boundary $r^{*}=R^{-}$,

$$
\left(u^{*}\right)^{-}=0 \text {, }
$$

where $\left[u^{*}\left(t^{*}, x^{*}, r^{*}\right)\right]^{ \pm},\left[v^{*}\left(t^{*}, x^{*}, r^{*}\right)\right]^{ \pm},\left[w^{*}\left(t^{*}, x^{*}, r^{*}\right)\right]^{ \pm}$ are denoted as the velocity components in the directions of $r^{*}, \theta$, and $x^{*}$, respectively, $\left[p^{* *}\left(t^{*}, x^{*}, r^{*}\right)\right]^{ \pm}$is the pressure, $r^{*}=R^{+}+\zeta^{*}\left(t^{*}, x^{*}\right)$ is the equation of the interface, $R^{-}$is the radius of the rigid cylindrical boundary, $D / D t^{*}$ is defined as the same form in (2)-(4), $T$ is the surface tension coefficient on the interface, and $S^{*}$ is the curvature of the interface,

$$
\begin{aligned}
S^{*}= & \frac{\partial^{2} \zeta^{*}}{\partial x^{* 2}}\left[1+\left(\frac{\partial \zeta^{*}}{\partial x^{*}}\right)^{2}\right]^{-3 / 2} \\
& -\left\{\left(R^{+}+\xi^{*}\right)\left[1+\left(\frac{\partial \xi^{*}}{\partial x^{*}}\right)^{2}\right]^{1 / 2}\right\}^{-1} .
\end{aligned}
$$

A uniform equilibrium state of (34)-(41) is

$$
\begin{aligned}
\left(u^{*}\right)^{ \pm}= & 0, \quad\left(v^{*}\right)^{ \pm}=0, \quad\left(w^{*}\right)^{ \pm}=c, \quad \zeta^{*}=0, \\
\left(p^{*}\right)^{+}= & {\left[\left(\omega^{*}\right)^{2} / 2\right]\left(\rho^{*}\right)^{+}\left(r^{*}\right)^{2}, } \\
\left(p^{*}\right)^{-}= & \left(\frac{\left(\omega^{*}\right)^{2}}{2}\right)\left(r^{*}\right)^{2}\left(\rho^{*}\right)^{-}-\left(\frac{T}{R^{+}}\right) \\
& -\left(\frac{\left(\omega^{*} R^{+}\right)^{2}}{2}\right)\left[\left(\rho^{*}\right)^{-}-\left(\rho^{*}\right)^{+}\right],
\end{aligned}
$$

where $c$ is a nonzero real constant and the wave speed of the flow.

To make all these variables nondimensional using appropriate units and a stretching parameter based upon the long-wave approximation, we let $\epsilon$ be a small parameter representing the ratio of a radial length scale to a longitudinal length scale and define

$$
\begin{aligned}
& (r, x)=\left(r^{*}, \epsilon^{1 / 2} x^{*}\right) / R^{+}, \quad t=2 \epsilon^{3 / 2} \omega^{*} t^{*}, \\
& \operatorname{Ro}=c /\left(2 \omega^{*} R^{+}\right), \\
& \left(u^{ \pm}, v^{ \pm}, w^{ \pm}\right) \\
& \quad=\left[\epsilon^{-1 / 2}\left(u^{*}\right)^{ \pm},\left(v^{*}\right)^{ \pm},\left(w^{*}\right)^{ \pm}\right] /\left(2 \omega^{*} R^{+}\right), \\
& p^{ \pm}=\left(p^{*}\right)^{ \pm} /\left[4 \rho_{0}\left(\omega^{*} R^{+}\right)^{2}\right],
\end{aligned}
$$

$\tau=\frac{T}{\left[4 \rho_{0}\left(\omega^{*}\right)^{2}\left(R^{+}\right)^{3}\right]}, \quad \rho^{ \pm}=\frac{\left(\rho^{*}\right)^{ \pm}}{\rho_{0}}$,

$\zeta=\frac{\zeta^{*}}{R^{+}}, \quad H=\frac{R^{-}}{R^{+}}$,

where Ro is the Rossby number and $\rho_{0}$ is the density scale. In terms of these variables, (34)-(41) become the following: in $0<r<1+\xi$ or $1+\zeta<r<H$ and $-\infty<x<\infty$,

$$
\begin{aligned}
& (r u)_{r}+(r w)_{x}=0 \\
& \epsilon^{2} u_{t}+\epsilon u u_{r}+\epsilon w u_{x}-r^{-1} v^{2}-v-(r / 4)=-\rho^{-1} p_{r} \\
& \epsilon v_{t}+u v_{r}+w v_{x}+r^{-1} u v+u=0 \\
& \epsilon w_{t}+u w_{r}+w w_{x}=-\rho^{-1} p_{x}
\end{aligned}
$$

and

$$
u, v, w, p, \rho= \begin{cases}u^{+}, v^{+}, w^{+}, p^{+}, \rho^{+}, & \text {for } 0<r<1+\zeta ; \\ u^{-}, v^{-}, w^{-}, p^{-}, \rho^{-}, & \text {for } 1+\zeta<r<H ;\end{cases}
$$

on the interface $r=1+\zeta$,

$$
\epsilon \xi_{t}+w^{ \pm} \xi_{x}-u^{ \pm}=0
$$




$$
\begin{aligned}
p^{-}-p^{+}= & \tau\left(\epsilon \zeta_{x x}\left[1+\epsilon\left(\zeta_{x}\right)^{2}\right]^{-3 / 2}\right. \\
& \left.-\left\{(1+\zeta)\left[1+\epsilon\left(\zeta_{x}\right)^{2}\right]^{1 / 2}\right\}^{-1}\right)
\end{aligned}
$$

at the boundary $r=H$,

$$
u^{-}=0 \text {. }
$$

Obviously a uniform equilibrium state of (42)-(48),

$$
\begin{aligned}
& u_{0}^{ \pm}=0, \quad v_{0}^{ \pm}=0, \quad w_{0}^{ \pm}=\mathrm{Ro}, \quad \zeta_{0}=0, \\
& p_{0}^{+}=\left(\frac{1}{8}\right) \rho^{+} r^{2}, \quad p_{0}^{-}=\left(\frac{1}{8}\right) r^{2} \rho^{-}-\tau-\left(\frac{1}{8}\right)\left(\rho^{-}-\rho^{+}\right) .
\end{aligned}
$$

In order to find the nontrivial solutions of (42)-(48), we let $\mathrm{Ro}=\lambda+\sigma \epsilon$ and expand $u, v, w, p, \zeta$ in an asymptotic series of the form

$$
f(t, x, r)=f_{0}(t, x, r)+\epsilon f_{1}(t, x, r)+\epsilon^{2} f_{2}(t, x, r)+\cdots,
$$

with $\epsilon$ small. The zeroth-order terms of $u, v, w, p$, and $\zeta$ are chosen as the equilibrium state (49) and (50). Substitution of the asymptotic series expansions of $u, v, w, p, \zeta$, and Ro into (42)-(48) yields a sequence of equations and boundary conditions for the successive approximations. We solve the successive approximate equations to have the successive-order approximations of the solutions of (42)(48). The first-order approximation is

$$
\begin{aligned}
& \left(r u_{1}^{ \pm}\right)_{r}+r w_{1 x}^{ \pm}=0, \\
& \rho^{ \pm} v_{1}^{ \pm}-p_{1 r}^{ \pm}=0, \\
& u_{1}^{ \pm}+\lambda v_{1 x}^{ \pm}=0, \\
& p_{1 x}^{ \pm}+\rho^{ \pm} \lambda w_{1 x}^{ \pm}=0 ;
\end{aligned}
$$

at $r=1$,

$$
\begin{aligned}
& u_{1}^{ \pm}-\lambda \zeta_{1 x}=0, \\
& p_{1}^{-}-p_{1}^{+}+\left[-\tau+\left(\rho^{-}-\rho^{+}\right) / 4\right] \xi_{1}=0
\end{aligned}
$$

at $r=H$,

$$
u_{1}^{-}=0 \text {. }
$$

This is a nonstandard eigenvalue problem with the eigenparameter $\lambda$. To have nontrivial solutions, we can show that $\lambda$ must satisfy the following equation:

$$
\begin{gathered}
\lambda\left(\rho^{-}-\rho^{+}\right) \Delta(1) J_{0}\left(\frac{1}{\lambda}\right)-\left(\frac{2 \lambda^{2} \rho^{-}}{\pi}\right) J_{1}\left(\frac{H}{\lambda}\right) \\
=\left(\tau-\frac{\left(\rho^{-}-\rho^{+}\right)}{4}\right) J_{1}\left(\frac{1}{\lambda}\right) \Delta(1),
\end{gathered}
$$

where

$$
\Delta(r)=J_{1}\left(\frac{r}{\lambda}\right) Y_{1}\left(\frac{H}{\lambda}\right)-J_{1}\left(\frac{H}{\lambda}\right) Y_{1}\left(\frac{r}{\lambda}\right)
$$

and $Y_{1}(z)$ is the Bessel function of the second kind of order unity. ${ }^{32}$ The critical values of $\lambda$ are determined by (58). It is also easy to show that $J_{1}(1 / \lambda) \neq 0$ and $\Delta(1) \neq 0$. If $\lambda=1 / \mu$, then $\mu$ must satisfy

$$
F_{0}(\mu) \equiv \mu\left\{\left(\rho^{-}-\rho^{+}\right) J_{0}(\mu)-\mu\left[\tau-\left(\rho^{-}-\rho^{+}\right) / 4\right] J_{1}(\mu)\right\}
$$

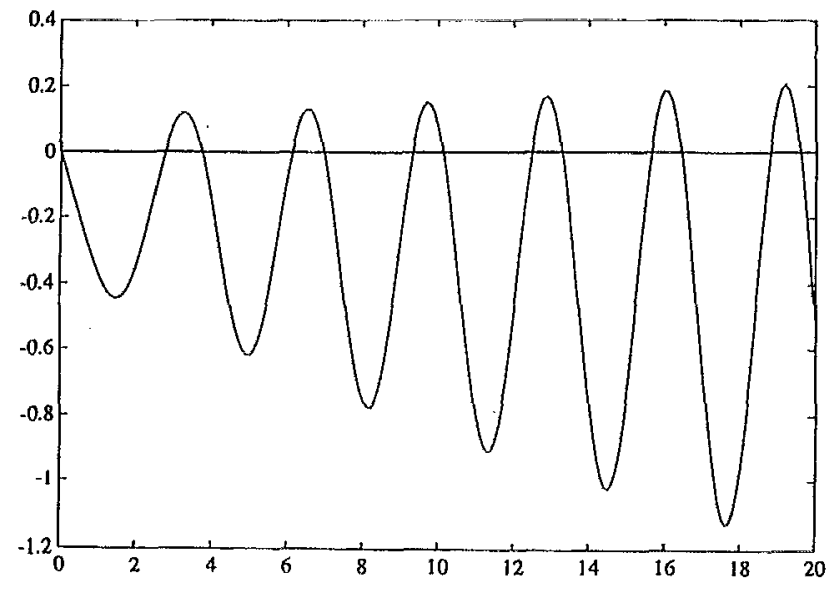

FIG. 4. The graph of function $F_{0}(\mu)$ for $0<\mu<20$ with $\rho^{-}=1, \rho^{-}=0.5$, and $\tau=1$.

$$
\begin{aligned}
& \times\left[J_{1}(\mu) Y_{1}(\mu H)-J_{1}(\mu H) Y_{1}(\mu)\right] \\
& -\left(2 \rho^{-} / \pi\right) J_{1}(\mu H)=0 .
\end{aligned}
$$

By using the asymptotic formulas of $J_{0}(z), J_{1}(z)$, and $Y_{1}(z)$, we can obtain that there are infinitely many positive roots, $0<\mu_{1}<\mu_{2}<\mu_{3}<\cdots \rightarrow+\infty$ for $F_{0}(\mu)=0$. The graph of function $F_{0}(\mu)$ is given in Fig. 4 , which also gives first several roots. Thus the critical value of $\lambda$ is $\lambda_{n}=1 / \mu_{n}$ with $n=1,2,3, \ldots$. In the following, $\lambda$ is chosen as $\lambda_{n}$ for some $n>0$.

If $\lambda=\lambda_{n}$, the nontrivial solution of (51)-(57) is

$$
\begin{aligned}
& u_{1}^{+}=\lambda_{n}\left(\frac{J_{1}\left(r / \lambda_{n}\right)}{J_{1}\left(1 / \lambda_{n}\right)}\right) \xi_{1 x}, \quad u_{1}^{-}=\lambda_{n}\left(\frac{\Delta(r)}{\Delta(1)}\right) \xi_{1 x}, \\
& v_{1}^{+}=-\left(\frac{J_{1}\left(r / \lambda_{n}\right)}{J_{1}\left(1 / \lambda_{n}\right)}\right) \xi_{1}, \quad v_{1}^{-}=-\left(\frac{\Delta(r)}{\Delta(1)}\right) \xi_{1}, \\
& w_{1}^{+}=\sigma-\left(\frac{J_{0}\left(r / \lambda_{n}\right)}{J_{1}\left(1 / \lambda_{n}\right)}\right) \xi_{1}, \quad w_{1}^{-}=\sigma-\left(\frac{\Delta_{0}(r)}{\Delta(1)}\right) \xi_{1}, \\
& p_{1}^{+}=\left(\frac{\lambda_{n} \rho^{+} J_{0}\left(r / \lambda_{n}\right)}{J_{1}\left(1 / \lambda_{n}\right)}\right) \zeta_{1}, \quad p_{1}^{-}=\left(\frac{\lambda_{n} \rho^{-} \Delta_{0}(r)}{\Delta(1)}\right) \xi_{1},
\end{aligned}
$$

where

$$
\Delta_{0}(r)=J_{0}\left(\frac{r}{\lambda_{n}}\right) Y_{1}\left(\frac{H}{\lambda_{n}}\right)-J_{1}\left(\frac{H}{\lambda_{n}}\right) Y_{0}\left(\frac{r}{\lambda_{n}}\right) .
$$

However, we still can not determine the function $\xi_{1}$ at this stage. In order to derive the equation for $\xi_{1}$, we have to use the second-order approximation of $(42)-(48)$, which is the following:

$$
\begin{aligned}
& \left(r u_{2}^{ \pm}\right)_{r}+\left(r w_{2}^{ \pm}\right)_{x}=0, \\
& \rho^{ \pm} v_{2}^{ \pm}-p_{2 r}^{ \pm}=\rho^{ \pm}\left[\lambda_{n} u_{1 x}^{ \pm}-r^{-1}\left(v_{1}^{ \pm}\right)^{2}\right], \\
& u_{2}^{ \pm}+\lambda_{n} v_{2 x}^{ \pm}=-v_{1 t}^{ \pm}-u_{1}^{ \pm} v_{1 r}^{ \pm}-w_{1}^{ \pm} v_{1 x}^{ \pm}-r^{-1} u_{1}^{ \pm} v_{1}^{ \pm}, \\
& p_{2 x}^{ \pm}+\rho^{ \pm} \lambda_{n} w_{2 x}^{ \pm}=\rho^{ \pm}\left(-w_{1 t}^{ \pm}-u_{1}^{ \pm} w_{1 r}^{ \pm}-w_{1}^{ \pm} w_{1 x}^{ \pm}\right) ;
\end{aligned}
$$

at $r=1$, 
$u_{2}^{ \pm}-\lambda_{n} \xi_{2 x}=\xi_{1 t}+w_{1}^{ \pm} \xi_{1 x}-u_{1 r}^{ \pm} \xi_{1}$,

$$
\begin{aligned}
& p_{2}^{-}-p_{2}^{+}+\left\{\left[\left(\rho^{-}-\rho^{+}\right) / 4\right]-\tau\right\} \xi_{2}=\left(\frac{1}{8}\right)\left(\rho^{+}-\rho^{-}\right) \xi_{1}^{2} \\
& +\zeta_{1}\left(p_{1 r}^{+}-p_{1 r}^{-}\right)+\tau\left(\xi_{1 x x}-\zeta_{1}^{2}\right)
\end{aligned}
$$

at $r=H$,

$$
u_{2}^{-}=0 \text {. }
$$

This is a nonhomogeneous boundary value problem and the right-hand sides are determined by $(61)-(64)$. Since $\lambda_{n}$ is an eigenvalue of the corresponding homogeneous problem (51)-(57), to find a solution of (66)-(72), the right-hand side of the equations must satisfy a solvability condition. From this condition an equation for $\xi_{1}(t, x)$ can be derived. After a straightforward but tedious calculation, we can obtain the following equation for $\zeta_{1}$ :

$$
B_{0} \xi_{1 t}+\sigma B_{0} \xi_{1 x}+B_{1} \xi_{1} \xi_{1 x}+B_{2} \xi_{1 x x x}=0,
$$

where

$$
\begin{aligned}
B_{0}= & \left(\frac{2}{\lambda_{n}}\right)\left[\rho^{+} \int_{0}^{1} J_{1}^{2}\left(\frac{r}{\lambda_{n}}\right) r d r J_{1}^{-2}\left(\frac{1}{\lambda_{n}}\right)\right. \\
& \left.+\rho^{-} \int_{1}^{H} \Delta^{2}(r) r d r \Delta^{-2}(1)\right] \\
& -\left(2 / \lambda_{n}\right)\left[\tau-\left(\rho^{-}-\rho^{+}\right) / 4\right], \\
B_{1}= & 3\left\{\rho^{-}\left[1+\Delta_{0}^{2}(1) \Delta^{-2}(1)\right]\right\} \\
& -\rho^{+}\left[1+J_{0}^{2}\left(1 / \lambda_{n}\right) J_{1}^{-2}\left(1 / \lambda_{n}\right)\right]-3 \tau, \\
B_{2}= & \tau-\lambda_{n}^{2}\left[\rho^{+} \int_{0}^{1} J_{1}^{2}\left(\frac{r}{\lambda_{n}}\right) r d r J_{1}^{-2}\left(\frac{1}{\lambda_{n}}\right)\right. \\
& \left.+\rho^{-} \int_{1}^{H} \Delta^{2}(r) r d r \Delta^{-2}(1)\right],
\end{aligned}
$$

where $\Delta(r)$ and $\Delta_{0}(r)$ are defined in (59) and (65), respectively. Now we shall prove the following claim.

Claim: $B_{0}$ is positive for all $n>1$.

Proof: By integration by parts and the properties of the Bessel functions, ${ }^{32}$

$$
\begin{aligned}
\int_{0}^{1} J_{1}^{2}\left(\frac{r}{\lambda_{n}}\right) r d r & =-\lambda_{n} J_{1}\left(\frac{1}{\lambda_{n}}\right) J_{0}\left(\frac{1}{\lambda_{n}}\right)+\int_{0}^{1} J_{0}^{2}\left(\frac{r}{\lambda_{n}}\right) r d r \\
& >-\lambda_{n} J_{1}\left(1 / \lambda_{n}\right) J_{0}\left(1 / \lambda_{n}\right)
\end{aligned}
$$

and

$$
\begin{aligned}
\int_{1}^{H} \Delta^{2}(r) r d r & =\lambda_{n} \Delta(1) \Delta_{0}(1)+\int_{1}^{H} r \Delta_{0}^{2}(r) d r \\
& >\lambda_{n} \Delta(1) \Delta_{0}(1) .
\end{aligned}
$$

From the definition of $B_{0}$ and (58),

$$
\begin{aligned}
B_{0}> & \left(\frac{2}{\lambda_{n}}\right)\left[\rho^{+}\left(-\lambda_{n}\right)\left(\frac{J_{0}\left(1 / \lambda_{n}\right)}{J_{1}\left(1 / \lambda_{n}\right)}\right)+\rho^{-} \lambda_{n} \Delta_{0}(1) \Delta^{-1}(1)\right] \\
& -\left(2 / \lambda_{n}\right)\left[\tau-\left(\rho^{-}-\rho^{+}\right) / 4\right]
\end{aligned}
$$

$$
\begin{aligned}
= & {\left[\left(\frac{2}{\lambda_{n} J_{1}\left(1 / \lambda_{n}\right)}\right)\right]\left[-\rho^{+} \lambda_{n} J_{0}\left(\frac{1}{\lambda_{n}}\right)\right.} \\
& +\rho^{-\lambda_{n} \Delta_{0}(1) \Delta^{-1}(1)} \\
& \left.\times J_{1}\left(\frac{1}{\lambda_{n}}\right)-\left(\tau-\frac{\left(\rho^{-}-\rho^{+}\right)}{4}\right) J_{1}\left(\frac{1}{\lambda_{n}}\right)\right]=0 .
\end{aligned}
$$

Thus $B_{0}$ is positive.

Therefore, by dividing both sides of (73) by $B_{0}$, we have the following $\mathrm{KdV}$ equation:

$$
\xi_{1 t}+\sigma \xi_{1 x}+\left(B_{1} / B_{0}\right) \zeta_{1} \zeta_{1 x}+\left(B_{2} / B_{0}\right) \xi_{1 x x x}=0,
$$

which has the same form as (31) in Sec. II. We can also obtain the traveling-wave solutions for (74) and the steady solitary wave solutions. However, the difference between (31) and (74) is that $B_{1}$ and $B_{2}$ in (74) may be zero, while those of (31) are never zero. If they are zero, then the KdV equation is no longer a right model equation, and different stretching parameters have to be introduced so that either modified $\mathrm{KdV}$ equation or a fifth-order perturbed $\mathrm{KdV}$ equation can be derived, as it has been done for the corresponding classical wave problems in a two-layer fluid. ${ }^{31}$ If we let $B_{1}$ and $B_{2}$ not be zero, then the steady solitary wave solution is

$$
\eta_{1}(x)=\left(-3 \sigma B_{0} / B_{1}\right) \operatorname{sech}^{2}\left[\left(-\sigma B_{2} / 4 B_{0}\right)^{1 / 2} x\right] .
$$

For $B_{2}<0, \sigma$ is positive and the solitary wave exists only in a supercritical flow. When $B_{2}>0, \sigma$ is negative and the wave of the flow is subcritical.

In the following we shall discuss some limiting cases from the general situation.

Case 1. $\tau=0$, i.e., no surface tension on the interface.

Then the critical speed is $\lambda_{n}=1 / \mu_{n}$ where $\mu_{n}$ satisfies

$$
\begin{gathered}
\left(\rho^{-}-\rho^{+}\right)\left[J_{0}(\mu)+(\mu / 4) J_{1}(\mu)\right]-\left(2 \rho^{-/} \pi \mu\right) J_{1}(\mu H) \\
\times\left[J_{1}(\mu) Y_{1}(\mu H)-J_{1}(\mu H) Y_{1}(\mu)\right]^{-1}=0 .
\end{gathered}
$$

From (73) and the claim, we can see that $B_{0}>0$ and $B_{2}<0$. By (58), $B_{1}$ can be simplified to

$$
\begin{aligned}
B_{1}= & 3\left(\rho^{-}-\rho^{+}+\left(\rho^{-}\right)^{-1}\left\{\rho^{+} J_{0}\left(\mu_{n}\right) J_{1}^{-1}\left(\mu_{n}\right)\right.\right. \\
& \left.\left.-\left[\left(\rho^{-}-\rho^{+}\right) \mu_{n} / 4\right]\right\}^{2}-\rho^{+}\left[J_{0}\left(\mu_{n}\right) / J_{1}\left(\mu_{n}\right)\right]^{2}\right) .
\end{aligned}
$$

If $\rho^{-}$and $\rho^{+}$are fixed with $\rho^{-}>\rho^{+}$, there exists some $H$ such that $B_{1}$ equals zero. In Fig. 5, we show the graph of $B_{1}$ vs $H$ with $n=1$ and can find that there are zero points of $B_{1}$ when $H$ changes. Such $H$ is called the critical radius. When $H$ is equal to the critical radius, the KdV equation is no longer valid for nonlinear theory and other stretching parameters must be introduced to have nonlinear model equations. ${ }^{31}$

Case 2. $\tau=0$ and $\rho^{+}=\rho^{-}$, i.e., a single-layer fluid in a long cylinder.

In this case, $B_{1}=0$ and we only have a linearized $\mathrm{KdV}$ equation. This problem was studied by Long $^{3}$ and Yih et $a l^{4}$ using the method of separation of variables and also considered by Grimshaw. ${ }^{30}$

Case 3. $\tau=0$ and $\rho^{+} \rightarrow 0$. 


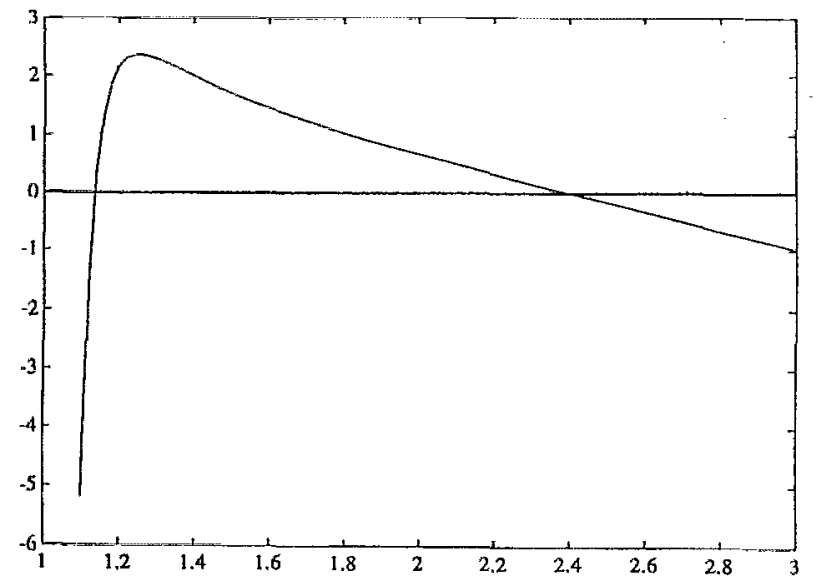

FIG. 5. The graph of $B_{1}$ vs $H$ for $1<H<3$ with $\rho^{-}=1, \rho^{+}=0.5$, and $n=1$.

In this limiting case, we can have the coefficients of the $\mathrm{KdV}$ equation as follows:

$$
\begin{aligned}
& B_{0}=\left(\frac{2 \rho^{-}}{\lambda_{n}}\right) \int_{1}^{H} \Delta^{2}(r) r d r \Delta^{-2}(1)+\left(\frac{\rho^{-}}{2 \lambda_{n}}\right), \\
& B_{1}=3 \rho^{-}\left[1+\Delta_{0}^{2}(1) / \Delta^{2}(1)\right], \\
& B_{2}=-\lambda_{n}^{2} \rho^{-} \int_{1}^{H} \Delta^{2}(r) r d r \Delta^{-2}(1),
\end{aligned}
$$

where $\Delta(r)$ and $\Delta_{0}(r)$ are defined in (59) and (65). The critical speed is $\lambda_{n}=1 / \mu_{n}$, where $\mu_{n}$ satisfies

$$
\begin{aligned}
\pi \mu & {\left[J_{0}(\mu)+(\mu / 4) J_{1}(\mu)\right]-2 J_{1}(\mu H)\left[J_{1}(\mu) Y_{1}(\mu H)\right.} \\
& \left.-J_{1}(\mu H) Y_{1}(\mu)\right]^{-1}=0 .
\end{aligned}
$$

Again, all $B_{0}, B_{1}$, and $B_{2}$ are not zero, which is similar to the case for a two-dimensional water wave problem. ${ }^{21}$ However, we still have infinitely many critical wave speeds, which is similar to the case for a two-dimensional stratified fluid. Therefore the rotating layer of fluid resembles the features from both a two-dimensional fluid of constant density and a stratified fluid.

Remark: It can be seen that the governing equations (42) - (48) can be considered as two sets of equations: one for the inner layer and another for the outer layer. The only equation connecting the two layers is (47). Therefore, if we consider a single rotating layer adjacent to the cylindrical wall, which means $\rho^{+}=0$ and is similar to a twodimensional fluid bounded below by a rigid bottom and above by a free surface, then we can let $u^{+}=v^{+}=w^{+}=p^{+}=0$ and solve the equations for the outer layer. The same formula as in Case 3 will be obtained with $\tau \neq 0$. However, a single-layer rotating fluid is different from that in Case 3. For a single-layer fluid, $u^{+}, v^{+}$, and $w^{+}$are undetermined, since it is meaningless to prescribe any velocity outside the fluid, while $u_{1}^{+}, v_{1}^{+}$, and $w_{1}^{+}$ in Case 3 are determined by (61)-(63) and are usually not zero. This can also be applied to the case in Sec. II if the necessary condition $\rho^{+}<\rho^{-}$in this section is omitted and $u^{-}=v^{-}=w^{-}=0$ and $p^{-}=P_{0}$ are assumed. Although it seems that the single-layer rotating fluid and the two-layer rotating fluid are related physically since the former may be considered a limiting case of the latter, these are also different mathematically. For a two-layer rotating fluid the velocities for the outer and inner layer are related to each other by the condition on the interface, and they must be solved simultaneously from the governing equations of outer- and inner-layer fluid, while for a single-layer fluid the velocity is exclusively determined by the equations in the fluid and the pressure outside the fluid. This is also true for single-layer and two-layer channel flows. ${ }^{27,31}$

\section{CONCLUSIONS}

In this paper, we have studied the weakly nonlinear theory for wave propagation in a rotating flow with free surface or a rotating two-fluid flow bounded by a rigid cylinder. If the flow is axisymmetric and rotating with an angular speed $\omega^{*}$ around the longitudinal axis, then there is a wave moving with constant speed $c$ and a nondimensional wave speed $\mathrm{Ro}=\left(c / 2 \omega^{*} R\right)$, the Rossby number, can be defined, where $R$ is the radius of the fluid at infinity for a single-layer fluid flow and the radius of the innerlayer fluid at infinity for a two-fluid flow. For the two-fluid flow with an interface between them, we assume that the density of the outer-layer fluid is greater than or equal to the density of the inner-layer fluid. It is found that there are infinitely many critical values of Ro for both cases and near each of them the KdV equation for the radial displacement of the free surface from an equilibrium state can be derived and solitary wave solutions are found. Therefore these flows are very similar to a two-dimensional flow with density stratification. The coefficients of the $\mathrm{KdV}$ equation for the single-layer fluid will never be zero. But the coefficients of the $\mathrm{KdV}$ equation for the two-fluid flow can be zero for some special ratio of the radius of the inner-layer fluid to the radius of the outer-layer fluid, which implies that the $\mathrm{KdV}$ equation is not a right model equation in this situation and other model equations should be derived. ${ }^{31}$ One limiting case that the density of the inner-layer fluid is zero is also considered, and the $\mathrm{KdV}$ equation is always valid because of the nonzero coefficients.

The results obtained here may have several physical applications. In the vortex breakdown problem, the existence of such solitary waves has been observed in experiments ${ }^{5,6}$ and also formally derived using long-wave approximations if the fluid is contained in a cylinder. ${ }^{8,9}$ However, if the problem is considered as a free boundary problem that is closer to a real situation, our results indicate that such solitary waves also exist and should be observed in experiments. This also applies to the crystal growth process in a zero-gravity environment. It is shown that there always exists a solitary wave moving at the free surface if the rotating liquid column is used to produce high-quality crystals, although it may be linearly stable using the linear stability analysis. ${ }^{15,16}$ Such wave movement at the free surface will definitely affect the growth of the crystals and impair the quality of the product. Therefore it is necessary to find a method to eliminate such waves before taking experiments in an orbital laboratory. In the 
process of a separation of mixtures with two fluids, it is known that the final state of the rotating mixture always has two parts: the lighter one is in the inner layer and the heavier one is in the outer layer. However, our results provide analytical evidence that the motion of the interface in the final state may not be steady, and there is a solitary wave at the interface moving with constant speed.

Finally, we note that for a two-dimensional stratified flow a solution of the exact equations, whose approximation is the solitary wave solution for the KdV equation, will have a small oscillatory tail at infinity when the wave speed is near a second critical value. This has been shown formally by Akylas and Grimshaw, ${ }^{26}$ numerically by Vanden-Broeck and Turner, ${ }^{25}$ and analytically by Sun and Shen. ${ }^{29}$ For the solitary waves in a rotating fluid, we shall expect that such oscillatory tails will also appear at infinity for the solutions of the exact equations when the Rossby number is near the second critical value.

${ }^{1}$ H. P. Greenspan, The Theory of Rotating Fluids (Cambridge U.P., New York, 1968).

${ }^{2}$ C.-S. Yih, Stratified Flows, 2nd ed. (Academic, New York, 1980).

${ }^{3}$ R. R. Long, "Steady motion around a symmetrical obstacle moving along the axis of a rotating liquid," J. Meteorol. 10, 197 (1953).

${ }^{4}$ C.-S. Yih, W. O'Dell, and W. R. Debler, "Prevention of stagnation zones in flows of a stratified or a rotating fluid," Proc. U.S. Natl. Congress Appl. Mech. 4, 1441 (1962).

${ }^{5} \mathrm{~J}$. K. Harvey, "Some observations of the vortex breakdown phenomenon," J. Fluid Mech. 14, 585 (1962).

${ }^{6} \mathrm{~W}$. G. Pritchard, "Solitary waves in rotating fluids," J. Fluid Mech. 42, 61 (1970).

${ }^{7}$ T. B. Benjamin, "Theory of the vortex breakdown phenomenon," J. Fluid Mech. 14, 593 (1962).

${ }^{8}$ T. B. Benjamin, "Some developments in the theory of vortex breakdown," J. Fluid Mech. 28, 65 (1967).

${ }^{9} \mathrm{~S}$. Leibovich, "Weakly non-linear waves in rotating fluids," J. Fluid Mech. 42, 803 (1970).

${ }^{10} \mathrm{H}$. B. Squire, "Analysis of the vortex breakdown phenomenon, Part I," Aeronautics Department, Imperial College, Report No. 102, 1960.

${ }^{11}$ S. Leibovich, "The structure of vortex breakdown," Annu. Rev. Fluid Mech. 10, 221 (1978).

${ }^{12}$ M. S. Berger, "Remarks on vortex breakdown," Mathematical Aspects of Vortex Dynamics, edited by R. E. Caflisch (SIAM, Philadelphia, PA, 1989), p. 171.

${ }^{13} \mathrm{~J}$. S. Turner, "The constraints imposed on tornado-like vortices by the top and bottom boundary conditions," J. Fluid Mech. 25, 377 (1966).

${ }^{14} \mathrm{C}$. H. Chun and W. Wuest, "Suppression of temperature oscillations of thermal Marangoni convection in a floating zone by superimposing of rotating flows," Acta Astron. 9, 225 (1982).

${ }^{15} \mathrm{H}$. F. Bauer, "Response of a spinning liquid column to axial excitation," Acta Mech. 77, 153 (1989).

${ }^{16} \mathrm{H}$. F. Bauer, "Axi-symmetric natural frequencies and response of a spinning liquid column under strong surface tension," Acta Mech. 90, 21 (1991).

${ }^{17}$ J. Gillis and K. S. Shuh, "Stability of a rotating liquid column," Phys. Fluids 5, 1149 (1962).

${ }^{18} \mathrm{~J}$. Gillis and B. Kaufmann, "The stability of a rotating viscous jet, ${ }^{22} \mathrm{Q}$. Appl. Math. 19, 301 (1962).

${ }^{19} \mathrm{H}$. P. Greenspan, "On centrifugal separation of a mixture," J. Fluid Mech. 127, 91 (1983).

${ }^{20} \mathrm{M}$. Ungarish, "Flow of a separating mixture in a rotating cylinder," Phys. Fluids 29, 640 (1986).

${ }^{21} \mathrm{D}$. J. Korteweg and G. deVries, "On the change of form of long waves advancing in a rectangular canal, and on a new type of long stationary waves," Philos. Mag. Ser. 5 39, 422 (1895).

${ }^{22} \mathrm{~A}$. S. Peters and Stoker, "Solitary waves in liquids having non-constant density," Commun. Pure Appl. Math. 13, 115 (1960).

${ }^{23} \mathrm{D}$. J. Benney, "Long non-linear waves in fiuid flows," J. Math. Phys. 45, 52 (1966).

${ }^{24}$ T. B. Benjamin, "Internal waves of finite amplitude and permanent form," J. Fluid Mech. 25, 241 (1966).

${ }^{25} \mathrm{~J} .-\mathrm{M}$. Vanden-Broeck and R. E. L. Turner, "Long periodic internal waves," Phys. Fluids A 4, 1929 (1992).

${ }^{26}$ T. R. Akylas and R. H. J. Grimshaw, "Solitary internal waves with oscillatory tails," J. Fluid Mech. 242, 279 (1992).

${ }^{27}$ A. M. Ter-Krikorov, "Thérie exacte des ondes longues stationnaires dans un liquide hétérogéne," J. Méc. 2, 351 (1963).

${ }^{28} \mathrm{~S}$. M. Sun and M. C. Shen, "Exact theory of solitary waves in a stratified fluid with surface tension, Part I. Nonoscillatory case," J. Diff. Eq. 105, 94 (1993).

${ }^{29}$ S. M. Sun and M. C. Shen, "Exact theory of solitary waves in a stratified fluid with surface tension, Part II. Oscillatory case," J. Diff. Eq. 105, 117 (1993).

${ }^{30}$ R. H. J. Grimshaw, "Resonant flow of a rotating fluid past an obstacle: The general case," Stud. Appl. Math. 83, 249 (1990).

${ }^{31}$ S. M. Sun and M. C. Shen, "Solitary waves in a two-layer fluid with surface tension," SIAM J. Math. Anal. 24, 866 (1993).

${ }^{32} \mathrm{G}$. N. Watson, A Treatise on the Theory of Bessel Functions, 2nd ed. (Macmillian, New York, 1945). 